\title{
Effects of drug abuse on oral health
}

\author{
Deepmala $^{1, *}$, Shweta Bali ${ }^{2}$, Meenu Taneja ${ }^{3}$, Priyanka $^{3}$ \\ ${ }^{1}$ PG Student, ${ }^{2}$ Professor \& HOD, ${ }^{3,4}$ Reader, Dept. of Periodontics and Oral Implantology, Santosh Dental College \& Hospital, \\ Ghaziabad, Uttar Pradesh, India
}

*Corresponding Author:

Email: deepmalamaids@gmail.com

\begin{abstract}
Drug abuse is one of major global concern among the social and medical ills of the twentieth century. Substance abuse ranks as one of the devastating and costly affairs in a present situation. These addictive drugs stimulating the brain, flooding it with neurotransmitters like dopamine which induces adaptive changes in the brain so that drug abuse may become compulsive for them. Thus drug abuse affect systemic as well as the oral health. This article discuss about the various drugs of abuse and their effect in the oral cavity along with the careful management of these addicts in the Dental clinic.
\end{abstract}

Keywords: Hallucinogen, Cannabis, Narcotics, Addiction, LXE.

\section{Introduction}

The use of illicit drug has increased worldwide. According to UNO (United nation office) on drug and crime 243 million people age (15-64yrs.) are users of illicit drug. ${ }^{1}$ Substance abuse is on rising trend more common in males then females. WHO (World Health Organization) estimated that 2 billion people consuming alcohol and 76.3 million people have complication related to alcohol abuse. Substance Abuse and mental Health Services Administration year 2000 survey report that addicts are physically and psychologically dependent on them and neglect their oral and systemic health and also reported that heavy alcohol drinking and illicit drug use is increasing worldwide and causes serious health problems. ${ }^{2}$ Addicts are physically as well as psychologically dependent on them. These addicts not only neglect their nutrition, health but as well as their oral health also. In addition to the direct effects of drugs on oral health drug abuse may aggravate oral problem indirectly through its adverse effects on user's behavior and lifestyle. Irregular eating pattern, poor oral hygiene, irregular dental visits and consumption of more sweet food affects their oral health. ${ }^{3}$

Systemic effects of substance abuse: Drug addiction has many deleterious effects systemically such as depression, anxiety, memory loss, various neuropsychological disorders and even death can occur because of internal bleeding or malignant hyperthermia. ${ }^{4}$

Oral Manifestation of Drug Abuse: Many of the abused substance have devastating consequences on oral health like mucosal ulceration dental Caries, tooth wear, meth mouth (Rampant dental caries), Xerostomia, Muscle Pain, Loss of sensation, grinding of teeth or bruxism. Cocaine can cause movement disorder manifest itself as transient chorea. Additionally buccolingual dyskinesia commonly known as "crack dancing" or boca torcida means twisted mouth can occur. In heroin abuse, increased numbers of decayed, missing and filled teeth can be detected in the mouth. Marijuana abuse can lead to acidic erosion of enamel caries due to cannabinoid hyper emesis in which frequent episodes of vomiting occur. These patients also develop dental caries, inflammation and hyperplasia of gingiva, uvulitis, leukoplakia, oral papilloma, and tongue carcinoma. Similarly high incidence of oral cancer, leucoedema, leucoplakia stomatitis, acute necrotizing ulcerative gingivitis, adult periodontitis is associated with increased use of tobacco. Effects of alcohol misuse has found to be noncarious tooth surface loss (Chemical Erosion), facial injury and mandible fracture, and addictive effects with tobacco increases 80 times risk of cancer in patients of alcohol abuse. ${ }^{5}$

Table 1: Commonly abused drug

\begin{tabular}{|l|l|}
\hline \multicolumn{1}{|c|}{ Substance abuse } & \multicolumn{1}{c|}{ Type } \\
\hline Narcotic Analgesic & $\begin{array}{l}\text { Opium, morphine, codeine, } \\
\text { Buprenorphine }\end{array}$ \\
\hline Stimulants & Amphetamine, cocaine \\
\hline Depressants & $\begin{array}{l}\text { Sedative-Hypnotics, } \\
\text { barbiturate, benzodiazepine }\end{array}$ \\
\hline Hallucinogens & $\begin{array}{l}\text { LSD(lysergic acid } \\
\text { diethylamide)oral tablets, } \\
\text { PSP(snorted or smoked) }\end{array}$ \\
\hline Cannabis & $\begin{array}{l}\text { Ganja, marijuana, hashish, } \\
\text { charas, hashish oil, bhang }\end{array}$ \\
\hline Others & $\begin{array}{l}\text { Tobacco,Alcohol, Heroin, } \\
\text { Ecstasy, Qat leaves }\end{array}$ \\
\hline
\end{tabular}

Effect of substance abuse on periodontal health: Drug addicts have high tendency for moderate to severe periodontitis characterized by loss of attachment. Addicts have high rate of plaque and calculus deposition resulting from oral hygiene neglect, xerostomia alteration in microbial profile. ${ }^{6}$ Alcohol abuse has been associated with increased risk of 
periodontal problems like gingival infection, increased pocket depth and loss of attachment. ${ }^{7}$ This occurs due to decreased resistance to infection by gram negative organisms during acute alcohol intoxication. ${ }^{8}$

Factors for Increase Orodental Diseases: Carbohydrate craving, use of carbohydrates additives in drug mix, masking of dental pain, poor nutrition, xerostomia, lack of dental hygiene. ${ }^{9}$

Factors Act as a Barrier to Care: Prolonged binging on drugs, Long waiting list for drug treatment and rehabilitation, Low self esteem or finance and poor acceptability of services. ${ }^{10}$

Oral Management Implications in Substance Abuse: Identify the dental patients with substance abuse by proper medical history, extra oral observation and clinical examination. Vital signs and needle tracks can be detected while taking blood pressure at each visit. Pain tolerance may be decreased in these patients. ${ }^{11}$ Patient may present with cutaneous manifestation i.e. infection and cellulites of the area where drug has been injected, delayed leg ulcer and nasal necrosis in cocaine abusers. Pop scars i.e. localized infection heals with characteristic irreversible scarring, gangrene of extremities can be present with cocaine abusers. ${ }^{12}$ Substance abusers have frequent missed appointment.

Facial or Oral Signs of Substance Abuse: Red face, blood shot eyes, enlarged parotid gland and breath odor are indicative of alcohol abuse. Periodontitis in adolescents is suggestive of marijuana abuse. Excessive bleeding during procedure, reduced salivary flow, large cervical caries (Rampant caries) on buccal surface are also indicators of patients with substance abuse.

Management of these Patients: There should be cautious use of local anesthesia in these patients also the use of vasoconstrictor should be limited during procedure. Non Steroid Anti Inflammatory Drugs (NSAIDs) for pain relieving should be used. For Alcoholic patients, acidic drugs example Aspirin and NSAIDs should be avoided. Non alcoholic mouth rinses should be prescribed and use of antibiotics should be limited in these patients. Postpone the treatment if drug is used within 24 hours of appointment. Corticosteroid can be used to reduce inflammation and swelling. Long acting anesthesia should be used for endodontic and surgical procedures. Comprehensive oral hygiene regime can be given like sialagogues, sugarless chewing gums. Oral moisturizer can be prescribed. Application of regular neutral fluorides is recommended and antifungal treatment should be given in subsequent visit.

Special care in pregnant women as substance Abuser: Substance used by pregnant women is a leading preventable cause of mental, physical and psychological problems in infant and children. Cautious use of local or general anesthesia and use of vasoconstrictor should be limited. NSAIDs should be used for pain relieving. For alcoholic patients acidic drugs should be avoided, non alcoholic rinses should be given. Minimum use of antibiotics and postpone the treatment if drug is used with in 24 hour.

\section{Discussion}

Drug abuse results in several direct consequences like multiple physical, mental and emotional problems. Medical problems includes cardiac arrest, respiratory depression, liver cirrhosis, nephropathy, infectious diseases such as tuberculosis, liver hepatitis AIDS, injury associated disability whereas mental disorder include depression. ${ }^{13}$

Medical complication that related to dentistry include abscesses at the injection site, viral hepatitis, Human Immunodeficiency Virus, endocarditis and anesthesia related problems. ${ }^{14}$

The long term effects of drugs abuse is the risk of developing permanent brain damage. In some case it may leads to death due to malignant hyperthermia, internal bleeding fatal overdosing and allergic reactions. ${ }^{15}$ As we all now oral health is a part of general health so health and wellbeing are likely to be improved by all efforts at oral health promotion. Rehabilitation center help these patients to take control of their lives and focus more on their health. Hence these centers can play an important role in changing patient's behavior. ${ }^{16}$ Improved access to dental health care via these centers might lead to change the lifestyle and behavior of the patients and in turn in improve the success of withdrawal treatment. So for these we have to come out with more oral health promotion programs at these centers and should prepare our self for such patients.

\section{Conclusion}

The problem of Substance abuse has emerged has a major issue in society and has become an area of major focus for healthcare professionals. All dental care providers can expect to see number of substance abusing patients. Extra caution has to be taken to minimize drug interactions, adverse effects while achieving this goal. Dentists should follow proper protocol while handling such kind of patients. Better dental care for patients who abuse illicit is needed to promote their oral health. Widespread education is needed to prepare our community to handle the demands. In addition collaboration between the social and health care sectors is required for their management.

\section{References}

1. Marques et al. The Impact of Oral Health Conditions Socioeconomic states and use of specific substance on quality of life of addictive person. BMC Oral health. 2015;15:38.

2. Saini GK, Gupta ND, Pra1bhat KC. Drug addiction and periodontal diseases. J Indian Soc Periodon. 2013;17:587-91.

3. Shekarchizadeh et al. Oral health behavior of drug addicts in withdrawal treatment. BMC Oral Health. 2013;13:11. 
4. Koyal. Illicit drug abuse affects periodontal health states. Saudi Med J. 2014;35:7.

5. Brand HS, Dun SN, Nieuw Amerongen AV. Ecstasy (MDMA) and oral health. Br Dent J. 2008;204:77-81.

6. Molendijk B, Ter Horst G, Kasbergen M, Truin GJ, Mulder J. Dental health in Dutch drug addicts. Community Dent Oral Epidemiol. 1996;24:117-19.

7. Tezal M, Grossi SG, Ho AW, Genco RJ. The effects alcohol consumption on periodontal disease. $J$ Periodontol. 2001;72(2):183-89

8. Marr JJ, Spilberg I. A mechanism for decreased resistance and infection by gram- negative organism during acute alcoholic intoxications. J Lab Clin Med. 1975;86:253-38.

9. National household survey on drug abuse 2000. Substance Abuse and Mental Health Data Archive (SAMHDA). Available as https://datafiles.samhsa.gov/study-dataset/nationalhousehold-survey-drug-abuse-2000-nhsda-2000-ds0001nid13706
10. Robinson P.G, Acquah S. Gibson B, Drug users: Oral health related attitude and behaviors. Briti Dent J. 2005; 198:219-24.

11. Scofield JC. The gravity of methamphetamine addiction. Dimen Dent Hyg. 2007;5:16-18.

12. Shekarchizadeh et al. Oral Health of Drug Abusers; A Review of Health Effects And Care. Iranian J Publ Health. 2013;42(9):929-40.

13. Meechan J.G. Drug abuser and dentistry. Dental Update. 1990;26:182-90.

14. Kraner J C, McCoy D J, Evans M A, Evans L E, Sweeney B J. Fatalities caused by the MDMA - related drug paramethoxy amphetamine (PMA). J Anal Taxicol. 2001;25;45-48.

15. Barbadoro P, Lucrezi D, Prospero E, Annino I. Improvement of knowledge, attitude and behavior about oral health in a population of alcohol addicted person. Alcohol. 2008;43:347-50. 